\title{
Real-world evidence on the strategy of olmesartan-based triple single-pill combination in Korean hypertensive patients: a prospective, multicenter, observational study (RESOLVE-PRO)
}

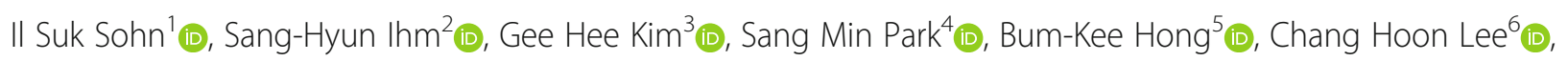

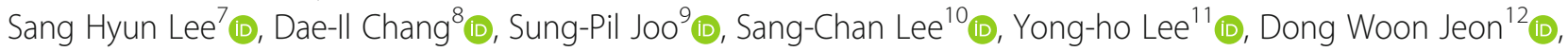
Kyung Tae Jung ${ }^{13}$, Si Jae Rhee ${ }^{14} \mathbb{D}$, Yoon-Jin $\mathrm{Cho}^{14}{ }_{\mathbb{D}}$, Chong-Jin Kim ${ }^{15^{*}}$ and Investigators

\begin{abstract}
Background: In this prospective, multicenter, non-comparative observational study, the effectiveness and safety of the triple single-pill combination (SPC) of olmesartan/amlodipine/hydrochlorothiazide (OM/AML/HCTZ) were evaluated in a real clinical practice setting in Korean patients with essential hypertension.

Methods: A total of 3752 patients were enrolled and followed for 12 months after administration of OM/AML/ HCTZ. Primary endpoint was change from baseline to month 6 in the mean systolic blood pressure (SBP). Secondary endpoints included changes from baseline in the mean SBP at month 3, 9, 12 and the mean diastolic blood pressure (DBP) at month 3, 6, 9, 12; changes in the mean SBP/DBP according to age and underlying risk factors; and blood pressure control rate (\%) at different time points. Adherence to and satisfaction with OM/AML/ HCTZ treatment among patients and physicians were assessed by medication possession ratio (MPR) and numeric rating scale, respectively, as exploratory endpoints. Safety was evaluated by the incidence and severity of adverse events (AEs) as well as the discontinuation rate due to AEs.
\end{abstract}

Results: OM/AML/HCTZ administration led to significant reductions in the mean SBP/DBP by 11.5/6.6, 12.3/7.0, 12.3/ 7.2, and 12.8/7.4 mmHg from baseline to month $3,6,9$ and 12 , respectively $(P<0.0001)$. The BP reductions were maintained throughout the 1-year observation period in all patients with different age groups and risk factors (diabetes mellitus, cardiovascular disease, and renal disease). The BP control rate (\%) of < 140/90 mmHg was 65.9, $67.9,68.9$, and $70.6 \%$ at month 3,6,9, and 12, respectively. The mean MPR during the observation period was 0.96 . The safety results were consistent with the previously reported safety profile of OM/AML/HCTZ.

\footnotetext{
*Correspondence: chongjinkim@naver.com

${ }^{15}$ Department of Cardiology, CHA Gangnam Medical Center, CHA University School of Medicine, Seoul, South Korea

Full list of author information is available at the end of the article
}

(c) The Author(s). 2021 Open Access This article is licensed under a Creative Commons Attribution 4.0 International License, which permits use, sharing, adaptation, distribution and reproduction in any medium or format, as long as you give appropriate credit to the original author(s) and the source, provide a link to the Creative Commons licence, and indicate if changes were made. The images or other third party material in this article are included in the article's Creative Commons licence, unless indicated otherwise in a credit line to the material. If material is not included in the article's Creative Commons licence and your intended use is not permitted by statutory regulation or exceeds the permitted use, you will need to obtain permission directly from the copyright holder. To view a copy of this licence, visit http://creativecommons.org/licenses/by/4.0/ The Creative Commons Public Domain Dedication waiver (http://creativecommons.org/publicdomain/zero/1.0/) applies to the data made available in this article, unless otherwise stated in a credit line to the data. 
Conclusions: Treatment with the triple SPC of OM/AML/HCTZ demonstrated significant effectiveness in reducing SBP/DBP and achieving target BP control with high adherence over the 1-year observation period in Korean hypertensive patients and was well-tolerated.

Trial registration: CRIS, KCT0002196, Registered 3 May 2016.

Keywords: Hypertension, Observational study, Olmesartan, Amlodipine, Hydrochlorothiazide, Real-world, Single-pill combination, Korea

\section{Background}

Hypertension is a critical public health burden affecting approximately 1.4 billion individual worldwide and wellknown significant risk factor for cardiovascular disease (CVD), such as myocardial infarction, stroke, and heart failure [1]. There is compelling evidence that treatment of hypertension reduces the risks of CVD, cerebrovascular disease, and all-cause mortality. However, despite the clear benefits of effective blood pressure (BP) control, the number of hypertensive patients in Korea has been increased to nearly 11 million, while the control rate of hypertension has remained suboptimal over a period of 10 years (44\% in 2007 vs. $41 \%$ in 2016) [2].

Lack of patient adherence to antihypertensive therapy has been considered as a major impediment to effective hypertension management. Multiple strategies have been developed to improve patient adherence and hence BP control. It has been well-known that combining antihypertensive agents from different classes produces a synergistic effect in reducing BP, which is estimated approximately five times more effective than increasing the dose of a single agent $[3,4]$. Reflecting this, recent hypertension guidelines have recommended the use of a single-pill combination (SPC) therapy to initiate antihypertensive therapy in most patients, with a preferred combination of Renin-Angiotensin System blocker and calcium channel blocker and/or thiazide/thiazide-like diuretic $[5,6]$. The triple SPC of olmesartan, amlodipine and hydrochlorothiazide (OM/AML/HCTZ) consists of the combination recommended by current guidelines and has shown to improve patient medication adherence compared with an extemporaneous combination (55.1\% vs. $15.9 \%, P<0.0001$, respectively) [7].

The efficacy and safety of the SPC of OM/AML/HCTZ have been previously determined in a number of randomized-controlled trials (RCTs), including 12-week TRINITY study, a global phase III clinical trial of OM/ AML/HCTZ, and 40-week open label extension of TRINITY study $[8,9]$. However, it is difficult to apply the results of the global studies to Korean patients in general as the proportion of Asian population included in those studies was small. Previous observational studies on OM/AML/HCTZ also lacked data on Asian ethnicity, particularly Koreans, and have been carried out over short periods $[10,11]$.
Real-world evidence (RWE) has been highlighted due to its ability to represent the actual clinical practice. Recently, RESOLVE study retrospectively investigated the effectiveness and safety of OM/AML/HCTZ in Korean hypertensive patients in a real clinical setting [12]. Since RWE of the use of triple SPC in Korean patients is still limited, this study prospectively evaluated the effectiveness and safety of OM/AML/HCTZ in Koreans patients with essential hypertension in a real-world practice in conjunction with the retrospective cohort study (RESOLVE-PRO).

\section{Methods}

\section{Study population}

Korean patients with essential hypertension who had initiated $\mathrm{OM} / \mathrm{AML} / \mathrm{HCTZ}$ at physician's discretion according to routine clinical considerations were included in this study. Neither prior treatment nor SPC treatment were restricted. Patients who were treatment-naïve were also included. Patients or their legal guardians consented to study participation after being informed about the protocol, collection of patient data, the effect, and possible adverse reactions (ADRs) of OM/AML/HCTZ. Patients were excluded if they had received OM/AML/HCTZ within 3 months prior to enrollment, if they were participating in another clinical study, or were deemed ineligible for this study at the discretion of the treating physician.

\section{Study design and procedures}

This study was a prospective, multicenter, noncomparative observational study to investigate the effectiveness and safety of OM/AML/HCTZ in a real clinical setting. Patient enrollment began on May 3, 2016 and the last follow-up was completed on January 28, 2019. A total of 70 investigators from 66 medical departments of 55 institutions participated in the study. BP was measured in a clinical setting of each institution in accordance with the standard measurement method of clinic BP from the 2018 Korea Society of Hypertension (KSH) guidelines [6]. This study was conducted in accordance with Declaration of Helsinki and the relevant pharmaceutical affairs law in Korea. Written approval of the study protocol and informed consent procedures were 
obtained from the Institutional Review Board (IRB) of all participating institutions.

Dose of OM/AML/HCTZ (20/5/12.5 mg, 40/5/12.5 mg and 40/10/12.5 mg are approved doses in Korea) and the frequency of follow-up were determined at the discretion of the treating physician. Apart from the routine 3month follow-up, the study involved no additional visits, treatments or procedures required beyond those occurring within the course of normal care.

Collected data included patient demographic characteristics, hypertension-related information, underlying diseases, concomitant drug use, medical history, and prescription information. Data on additional antihypertensive medications or dose escalation of OM/AML/HCTZ in patients with inadequate BP control were also collected.

\section{Effectiveness evaluation}

The effectiveness analysis set included patients who met enrollment criteria and received follow-up assessment at least once during the observation period after administration of $\mathrm{OM} / \mathrm{AML} / \mathrm{HCTZ}$ at baseline visit. The safety analysis set included patients who had administered $\mathrm{OM} / \mathrm{AML} / \mathrm{HCTZ}$ at least once during the observation period.

The primary endpoint was the change from baseline in the mean systolic BP (SBP) at month 6 following administration of OM/AML/HCTZ. The secondary endpoints included the changes in the mean SBP at month 3, 9, 12 and the mean diastolic BP (DBP) at month 3, 6, 9, 12 compared to baseline; the changes in the mean SBP/ DBP according to age and accompanying risk factors; and the rate of $\mathrm{BP}$ control at each visit. The rate of BP control was defined as the proportion of patients who had achieved SBP/DBP below the target of $<140 / 90$ $\mathrm{mmHg}$ during the observation period. The target $\mathrm{BP}$ goal of $\mathrm{SBP} / \mathrm{DBP}<140 / 90 \mathrm{mmHg}$ was set according to $2018 \mathrm{KSH}$ and 2018 European Society of Cardiology (ESC)/European Society of Hypertension (ESH) guidelines at the time of analysis $[5,6]$.

For exploratory endpoints, patient medication adherence was assessed using medication possession ratio (MPR). The MPR was calculated as a proportion, representing the number of days covered by prescription of $\mathrm{OM} / \mathrm{AML} / \mathrm{HCTZ}$, divided by the number of days between the date of the first prescription and the date for the last prescription. In addition, patients' and physicians' satisfaction on the use of OM/AML/HCTZ at month 6 and 12 were measured using an eleven-point numeric rating scale (NRS) (ranging from $0=$ 'not satisfied at all' to $10=$ 'completely satisfied').

\section{Safety evaluation}

Safety evaluation of OM/AML/HCTZ included the incidence and severity of adverse events (AEs) and ADRs, as well as the discontinuation rate of OM/AML/HCTZ due to AEs.

\section{Statistical analysis}

Unless specified otherwise, two-sided tests were used for all statistical analyses using SAS ver. 9.4 (SAS Institute, Cary, NC, USA), with a level of significance level set at $5 \%(P<0.05)$. Descriptive statistics including number of participants, mean and standard deviation were used for continuous data, while categorical data were presented using frequency and ratio (\%). The distributions of all continuous variables were tested for normality; parametric tests were performed for variables with a normal distribution, whereas non-parametric tests were for those without. The normality of data distribution was examined using the Shapiro-Wilk test with a $P$-value less than $5 \%(P<0.05)$. Paired $\mathrm{t}$-tests were performed for variables with a normal distribution, while Wilcoxon signed-rank tests and McNemar's tests were performed for those without. $P$-values were presented for these variables. No adjustments were made for missing data from continuous variables at particular time points; missing data due to patient dropout prior to completion of the study; and missing data from the safety analysis set.

\section{Results \\ Study population}

A total of 3752 patients were enrolled from 55 institutions between May 2016 and January 2019. Of the patients enrolled, 3687 patients $(98.3 \%)$ had administered $\mathrm{OM} / \mathrm{AML} / \mathrm{HCTZ}$ and 2468 patients $(65.8 \%)$ completed the study. The effectiveness analysis set included 3052 patients $(81.3 \%)$ and the safety analysis set included 3370 patients (89.8\%) (Fig. 1).

The patient demographic characteristics are summarized in Table 1. The mean age of the enrolled patients was $62.7 \pm 13.3$ years and $49.2 \%$ were aged 65 years or older. A total of $57.2 \%$ of them were male and $41.4 \%$ were female. The mean height, body weight, and body mass index were $163.0 \pm 9.3 \mathrm{~cm}, 70.1 \pm 14.0 \mathrm{~kg}$, and $26.1 \pm 4.1 \mathrm{~kg} / \mathrm{m}^{2}$, respectively. The mean duration of hypertension was $9.3 \pm 8.1$ years. The mean SBP and DBP at baseline were $143.6 \pm 19.2 \mathrm{mmHg}$ and $83.6 \pm$ $14.0 \mathrm{mmHg}$, respectively. Of the enrolled patients, $17.7 \%$ $(n=663)$ were smokers, 28.1\% $(n=1053)$ consumed alcohol, and $19.5 \%(n=731)$ had a family history of hypertension. A total of $63.4 \% \quad(n=2378)$ of the enrolled patients had underlying risk factors including others $(n=1597)$, diabetes mellitus (DM; $n=1231)$, and renal disease (RD; $n=141)$ (Table 1).

\section{Effectiveness}

In the effectiveness analysis set $(n=3052)$, the mean SBP was significantly reduced by $12.3 \pm 20.8 \mathrm{mmHg}$ from 


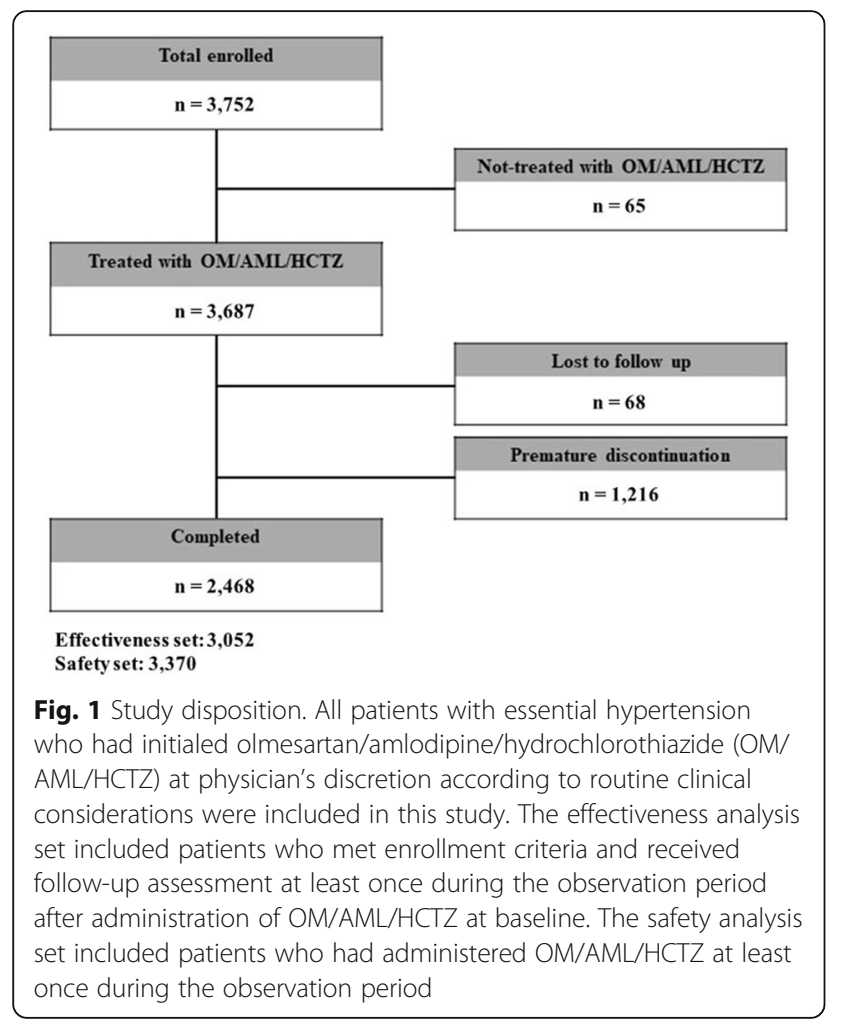

baseline $(143.3 \pm 19.4 \mathrm{mmHg})$ to 6 months $(130.6 \pm 16.1$ $\mathrm{mmHg})$ after administration of OM/AML/HCTZ $(P<$ $0.0001)$. The reductions in the mean SBP at month 3,9 and 12 were as follows: $11.5 \pm 20.4 \mathrm{mmHg}, 12.3 \pm 20.7$ $\mathrm{mmHg}$, and $12.8 \pm 20.4 \mathrm{mmHg}$, respectively (all $\mathrm{P}<$ $0.0001)$. The mean baseline DBP was also significantly reduced from baseline $(83.4 \pm 13.9 \mathrm{mmHg})$ after administration of $\mathrm{OM} / \mathrm{AML} / \mathrm{HCTZ}$ by $6.6 \pm 13.9 \mathrm{mmHg}, 7.0 \pm$ $13.9 \mathrm{mmHg}, 7.2 \pm 13.8 \mathrm{mmHg}$, and $7.4 \pm 13.6 \mathrm{mmHg}$ at month 3, 6, 9, and 12, respectively (all $P$-value $<0.0001$ ) (Fig. 2). The mean SBP/DBP was reduced to $132.1 / 76.8$ mmHg at month 3; $130.6 / 75.8 \mathrm{mmHg}$ at month 6; $130.0 / 75.3 \mathrm{mmHg}$ at month 9 ; and $129.1 / 75.0 \mathrm{mmHg}$ at month 12, respectively (Fig. 3).

The BP control rate of $<140 / 90 \mathrm{mmHg}$ at month 3, 6, 9, and 12 after the administration of OM/AML/HCTZ was 65.9, 67.9, 68.9, and 70.6\%, respectively (Fig. 4a). The mean MPR during the observation period was 0.96. NRS scores of patients' and physician's satisfaction with the use of SPC of OM/AML/HCTZ were 8.2 and 8.2 points at month 6; and 8.6 and 8.4 points at month 12 , respectively.

\section{Subgroup analysis}

In a subgroup analysis, the BP lowering effect of OM/ AML/HCTZ was evaluated in 1724 patients who had maintained the baseline dose of OM/AML/HCTZ throughout the entire study period, without additional antihypertensive medications to control BP. The mean SBP
Table 1 Demographic and baseline characteristics (enrolled set)

\begin{tabular}{|c|c|}
\hline Characteristic & Enrolled set $(n=3752)$ \\
\hline Age (yr) & $62.7 \pm 13.3$ \\
\hline \multicolumn{2}{|l|}{ Age group (yr) } \\
\hline$<60$ & $1395(37.2)$ \\
\hline $60-64$ & $458(12.2)$ \\
\hline $65-69$ & $552(14.7)$ \\
\hline $70-74$ & $548(14.6)$ \\
\hline $75-80$ & $499(13.3)$ \\
\hline$>80$ & $247(6.6)$ \\
\hline Elderly age ( $\geq 65 \mathrm{yr}$ ) & $1846(49.2)$ \\
\hline \multicolumn{2}{|l|}{ Sex } \\
\hline Male & $2145(57.2)$ \\
\hline Female & $1554(41.4)$ \\
\hline Baseline height $(\mathrm{cm}), n=2917$ & $163.0 \pm 9.3$ \\
\hline Baseline body weight $(\mathrm{kg}), n=2931$ & $70.1 \pm 14.0$ \\
\hline BMI $\left(\mathrm{kg} / \mathrm{m}^{2}\right), n=2877$ & $26.1 \pm 4.1$ \\
\hline Baseline SBP $(\mathrm{mmHg}), n=3640$ & $143.6 \pm 19.2$ \\
\hline Baseline DBP $(\mathrm{mmHg}), n=3638$ & $83.6 \pm 14.0$ \\
\hline Hypertension period (yr), $n=2603$ & $9.3 \pm 8.1$ \\
\hline Smoking status, smoker & $663(17.7)$ \\
\hline Alcohol consumption, yes & $1053(28.1)$ \\
\hline Family history of hypertension, yes & $731(19.5)$ \\
\hline Accompanying risk factor, yes & $2378(63.4)$ \\
\hline \multicolumn{2}{|l|}{ Accompanying risk factor } \\
\hline Renal disease & $141(3.8)$ \\
\hline Diabetes & $1231(32.8)$ \\
\hline Others & $1597(42.6)$ \\
\hline
\end{tabular}

Data are presented as mean \pm standard deviation or number (\%). Hypertension period $(\mathrm{mo})=($ enrollment date - date of hypertension diagnosis +1$) / 30.44$ The date of hypertension diagnosis was assumed to be the first day of the month in the recorded year. Duplicates were allowed for accompanying risk factors

$B M I$ body mass index, SBP systolic blood pressure, $D B P$ diastolic blood pressure

was significantly reduced at all time points from baseline $(142.1 \pm 18.8 \mathrm{mmHg})$ by $13.3 \pm 20.5 \mathrm{mmHg}, 13.9 \pm 20.3$ $\mathrm{mmHg}, \quad 13.3 \pm 20.2 \mathrm{mmHg}$, and $13.6 \pm 19.6 \mathrm{mmHg}$ at month 3, 6, 9, and 12, respectively. The mean SBP in the subgroup of patients at each month was as follows: $128.8 \pm 14.8 \mathrm{mmHg}, \quad 127.7 \pm 14.3 \mathrm{mmHg}, \quad 127.8 \pm 13.9$ $\mathrm{mmHg}$, and $127.4 \pm 13.6 \mathrm{mmHg}$, respectively (all $P<$ 0.0001) (Fig. S1). The mean baseline DBP was also significantly reduced at all time points by $6.9 \pm 13.2 \mathrm{mmHg}$, $7.4 \pm 13.5 \mathrm{mmHg}, 7.2 \pm 13.1 \mathrm{mmHg}$, and $7.5 \pm 12.9 \mathrm{mmHg}$ at month 3, 6, 9 and 12 from baseline $(82.8 \pm 13.7 \mathrm{mmHg})$. The mean DBP at each month was as follows: $75.8 \pm 10.3$ $\mathrm{mmHg}, 74.9 \pm 10.1 \mathrm{mmHg}, 74.7 \pm 10.0 \mathrm{mmHg}$, and $74.5 \pm$ $10.2 \mathrm{mmHg}$, respectively (all $P<0.0001$ ) (Fig. S1). In addition, the overall BP control rate of this subgroup of patients $(n=1724)$ was $71.2,73.1,71.6$, and $74.6 \%$ at 


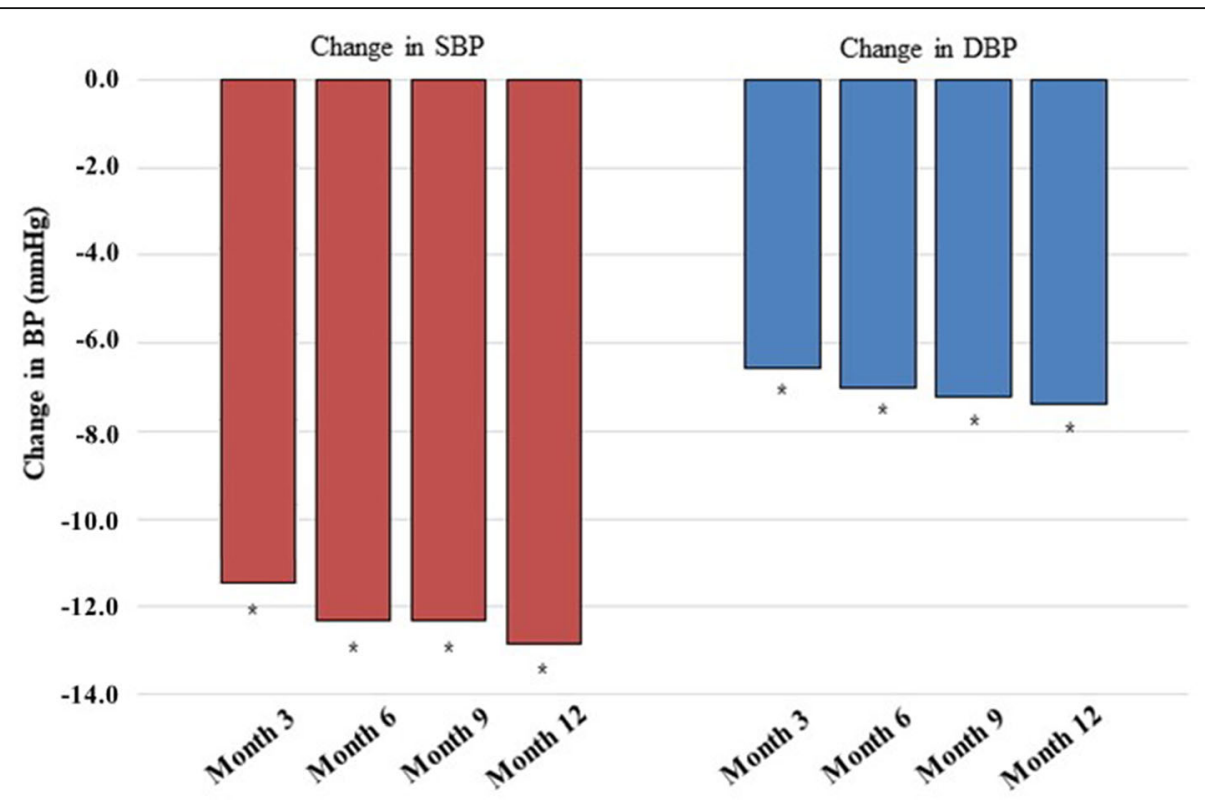

Fig. 2 Changes from baseline in the mean SBP/DBP at month 3, 6, 9, and 12 after olmesartan/amlodipine/hydrochlorothiazide (OM/AML/HCTZ) administration. Effectiveness set $(n=3052)$. BP, blood pressure; SBP, systolic BP; DBP, diastolic BP. ${ }^{*} P<0.0001$ (Wilcoxon singed-rank test)

month 3, 6, 9 and 12, respectively (Fig. 4b). The BP control rate achieved by each strength of OM/AML/HCTZ in the subgroup are as follows: $74.7,76.6,73.1$, and $76.8 \%$ at month $3,6,9$, and 12 in $20 / 5 / 12.5 \mathrm{mg}$ group $(n=1266)$; $60.6,65.7,67.6$, and $68.1 \%$ at month $3,6,9$, and 12 in $40 /$ $5 / 12.5 \mathrm{mg}$ group $(n=283) ; 62.5,59.6,66.4$, and $68.2 \%$ at month $3,6,9$, and 12 in 40/10/12.5 mg group, respectively (all $P<0.0001$ ) (Fig. 4b).
Moreover, the change in the mean SBP/DBP relative to the baseline following $\mathrm{OM} / \mathrm{AML} / \mathrm{HCTZ}$ administration was examined in patients with DM $(n=983)$, CVD $(n=509)$, or RD $(n=128)$. The patients in the DM and CVD groups showed significant reductions in the mean SBP/DBP at month $3,6,9$, and 12 , relative to the baseline (all $P<0.0001$ ). Moreover, the patients with $\mathrm{RD}$ showed significant reductions in SBP at all measured

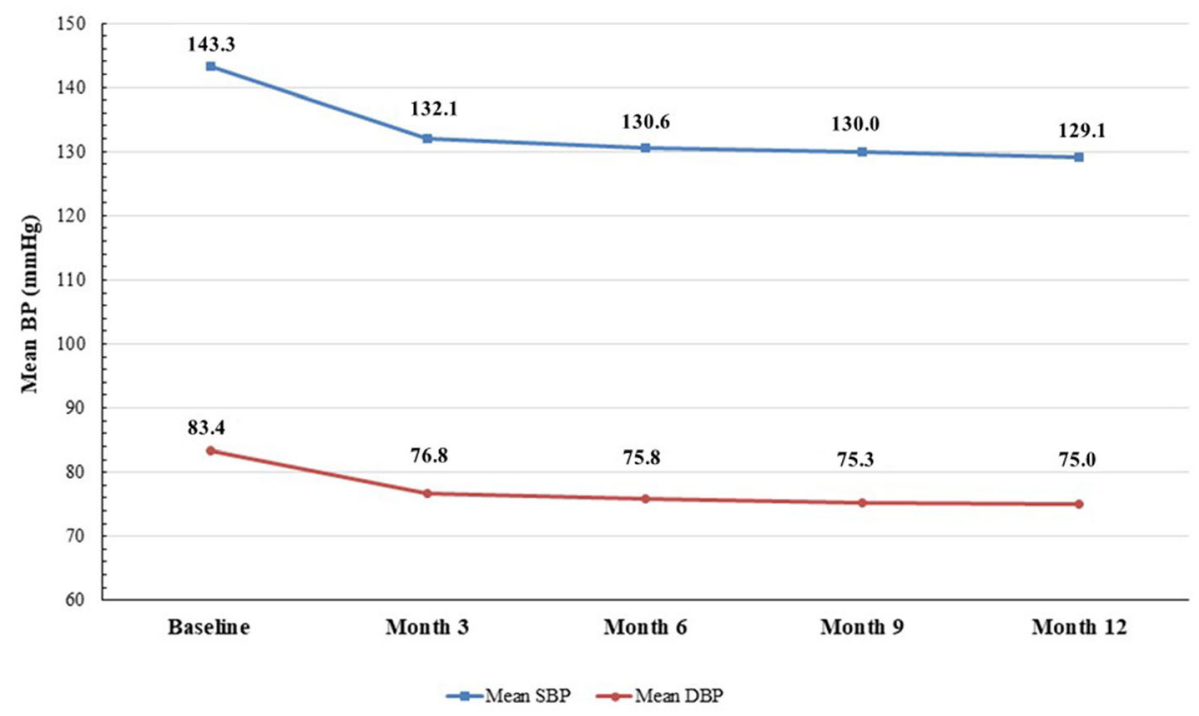

Fig. 3 Time course change from baseline in the mean SBP/DBP at month 3, 6, 9, and 12 after olmesartan/amlodipine/hydrochlorothiazide (OM/ AML/HCTZ) administration. Effectiveness set $(n=3052)$. BP, blood pressure; SBP, systolic BP; DBP, diastolic BP 

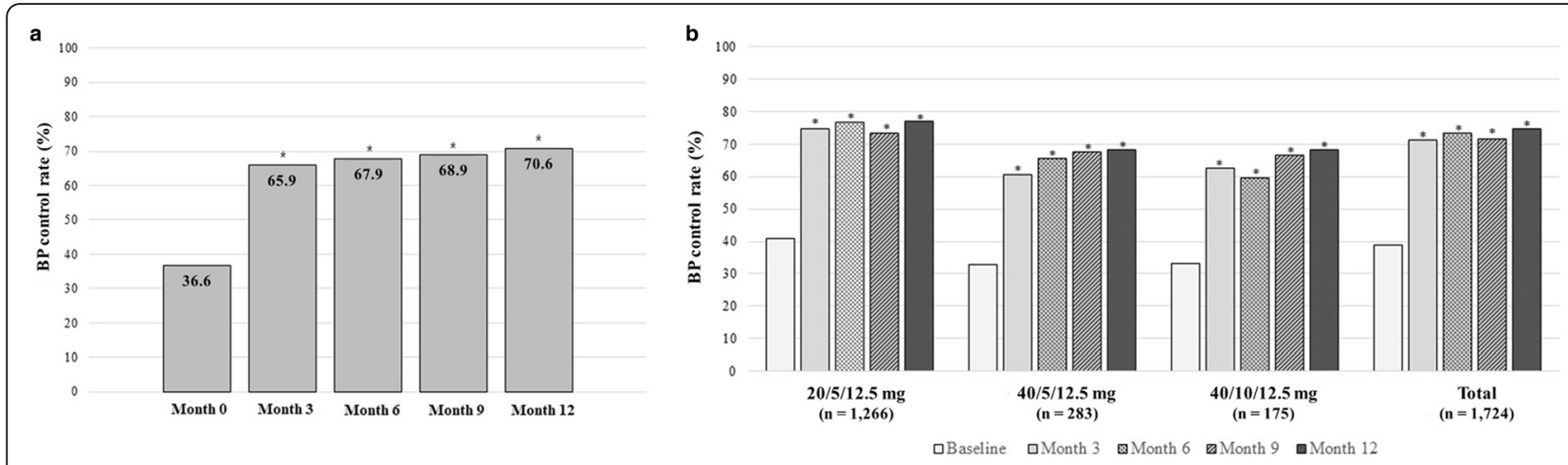

Fig. 4 Blood pressure (BP) control rates. a BP control rates at month 3, 6, 9, and 12 after olmesartan/amlodipine/hydrochlorothiazide (OM/AML/ HCTZ) administration in overall patients. b BP control rates at month 3, 6, 9, and 12 in patients treated only with OM/AML/HCTZ. Target BP, SBP/ DBP $<140 / 90 \mathrm{mmHg}$ ). Effectiveness set $(n=3052)$. BP, blood pressure; SBP, systolic BP; DBP, diastolic BP. ${ }^{*} P<0.0001$ (McNemar's test)

points during the study period with significant reductions in DBP observed at month 3 and 12, relative to the baseline SBP (all $P<0.0001$ ) (Table S1).

The change in the mean SBP/DBP relative to the baseline after OM/AML/HCTZ administration was examined according to age groups ( $<60$ years, $60 \leq \&<65$ years, $65 \leq \&<70$ years, $70 \leq \&<74$ years, $75 \leq \&<80$ years, and $>80$ years). Significant reductions in the mean SBP/DBP were observed at all measured points in all age groups $(P<0.0001$ to 0.0003$)$ (Table $\mathrm{S} 2)$.

\section{Safety assessment}

In the safety analysis set $(n=3370)$, AEs and serious AEs (SAEs) were reported by $26.8 \%(n=902)$ and $4.8 \%(n=$ 163 ), respectively (Table 2). Dizziness was the most frequently reported $\mathrm{AE}(n=183,5.4 \%)$, followed by headache $(n=55,1.6 \%)$, and arthralgia $(n=41,1.2 \%)$. SAEs included angina pectoris $(n=10,0.3 \%)$, pneumonia and chest pain $(n=8,0.2 \%$, for each SAE), and dizziness $(n=$ $5,0.2 \%)$.

The discontinuation rate of OM/AML/HCTZ due to AEs in the safety analysis set was reported at 7.6\% $(n=$ $255)$ with dizziness being the most common $(n=87$, $2.6 \%)$, followed by hypotension $(n=45,1.3 \%)$ (Table 2$)$. AEs that led to five deaths $(0.2 \%)$ were not considered to be related to OM/AML/HCTZ treatment. All deaths resulted from non-cardiovascular causes including gastric cancer, malignant lung neoplasm, small-intestine

Table 2 Summary of TEAE and ADR (safety set, $n=3370$ )

\begin{tabular}{lll}
\hline Variable & TEAE & ADR \\
\hline Total & $902(26.8)[1727]$ & $332(9.9)[626]$ \\
Serious & $163(4.8)[225]$ & $11(0.3)[14]$ \\
Leading to drug withdrawal & $255(7.6)[307]$ & $185(5.5)[215]$ \\
Leading to death & $5(0.2)[7]$ & $0[0]$ \\
\hline
\end{tabular}

Data are presented as number (\%) [case]

TEAE treatment-emergent adverse event, $A D R$ adverse drug reaction carcinoma, pneumonia, septic shock, cerebrovascular accident and renal failure ( $n=1$, for each death).

ADRs with causal relationship to OM/AML/HCTZ were reported by $9.9 \%(n=332)$. The most common ADR was dizziness $(n=106,3.2 \%)$, followed by hypotension $(n=56,1.7 \%)$. Serious ADRs (SADRs) were reported by $0.3 \%(n=11)$ (Table 2$)$. Of the 14 cases of SADRs, ten cases of SADRs that were categorized as 'unclassified' have remained at OM/AML/HCT treatment and were later reported as 'recovered'. Four cases of SADRs including chest pain, hypotension, dizziness, and hyponatremia led to discontinuation of OM/AML/ HCTZ treatment and were later reported as 'recovered.'

The discontinuation rate of OM/AML/HCTZ due to ADRs was reported by $5.5 \%(n=185)$. The most frequent ADRs causing withdrawal from the treatment were dizziness $(n=75,2.2 \%)$, followed by hypotension $(n=45,1.3 \%)$, and headache $(n=9,0.3 \%)$. No ADRrelated deaths occurred (Table 2).

\section{Discussion}

This study prospectively evaluated the effectiveness and safety of the triple SPC of OM/AML/HCTZ in Korean hypertensive patients in a real clinical practice setting.

Following administration of OM/AML/HCTZ, significant reductions from baseline in the mean SBP/DBP were observed at all measured time points (month 3, 6, 9 , and 12). The greatest reductions in both SBP and DBP were observed at month 3 and the reduced mean SBP/DBP was sustained close to $<130 / 80 \mathrm{mmHg}$ throughout the 12-month observation period. These results were in accordance with the recommendations of current ESC/ESH hypertension guideline, where the BP goal of at least $130 / 80 \mathrm{mmHg}$ is recommended in most patients, once SBP/DBP was safely controlled under the threshold of $140 / 90 \mathrm{mmHg}$ [5]. The significant BP reductions were consistently observed across all age groups and risk factors (Tables S1, S2). 
It is known that early and fast BP management is associated with more effective and lasting BP control and hence greater long-term clinical benefits. Previous studies have shown that achievement of BP control within 6 months is associated with significantly reduced incidence of cardiovascular outcomes [13]. In this study, OM/ AML/HCTZ demonstrated a fast onset and long-lasting effect on BP control by achieving the control rate of $65.9 \%$ at month 3 , which gradually increased to $70.6 \%$ until month 12 (Fig. 4a). Higher BP control rates were observed in the subgroup of patients (71.2 to 74.6\%), further supporting the effectiveness of OM/AML/HCTZ for controlling BP without dose escalation or addition of other agents (Fig. 4b). These results were comparable to that reported in 12-week TRINITY study (70.0\%) and in 40-week TRINITY-extension study (44.5 to $79.8 \%$, depending on treatment dose) [8, 9]. According to 2018 $\mathrm{KSH}$ guidelines, $71 \%$ of patients treated with antihypertensive therapy achieved BP control and this was supported by the present real-world study [6].

Patient satisfaction to treatment is highly correlated with patient adherence and treatment success. In this study, patients indicated high levels of satisfaction with and adherence to OM/AML/HCTZ treatment (NRS of 8.2 to 8.6 and MPR of 0.96 , respectively) which were possibly attributable to effective BP management throughout the treatment course.

Most hypertensive patients require more than single antihypertensive agent to control $\mathrm{BP}$ and often require concurrent use of three agents from different classes [3, $4]$. In Korea, it appears that approximately $60 \%$ of patients take two or more antihypertensive medications and $17.7 \%$ of them are receiving three or more different classes [2]. Several SPCs have been released to improve medication adherence by reducing pill burden and complexity of dosing regimens, and have shown to increase the $\mathrm{BP}$ control rate $[1,14,15]$. OM/AML/HCTZ was the first triple SPC launched in Korea and the efficacy of the triple SPC of OM/AML/HCTZ has been welldemonstrated in previous RCTs [8-10].

RCTs have limitations with respect to representing a wide diversity of patients in the real clinical practice, although it is regarded as the most reliable research design owing to its strict patient selection criteria and blinding process. In real-world, the hypertension status varies according to race/ethnicity, however, only limited data on the Korean population have been available. In addition, since most RCTs are conducted over a short period of up to 3 to 6 months, the long-term effect of a treatment often remains to be determined. This study was conducted prospectively in a routine clinical setting over a period of 12 months, involving a wide variety of patient groups. Thus, it was less prone to have recall bias compared with retrospective studies.
The subgroup analysis of this real-world study revealed that the majority of patients $(74.4 \%)$ had administered OM/AML/HCTZ at the standard dose of 20/5/ $12.5 \mathrm{mg}$. These patients were maintained on the same dose until month 12 and achieved the BP control rates greater than that reported in the previous global study where the patients were treated with the high dose of OM/AML/HCTZ 40/10/25 mg (73.1 to $76.8 \%$ vs. $69.9 \%$, respectively) [8]. Furthermore, according to the study, which examined the long-term efficacy of OM/AML/ HCTZ, the highest BP control rate at week 52 was achieved in the patients receiving 40/5/12.5 $\mathrm{mg}$ (79.8\%) [9]. Taken together, for Korean patients in real-world practice, the standard dose of OM/AML/HCTZ (20/5/ $12.5 \mathrm{mg}$ ) was as effective option as the high dose OM/ AML/HCTZ.

The present study was initially designed according to the 2014 Joint National Committee volume 8. During the study process, $2018 \mathrm{KSH}$ and $2018 \mathrm{ESC} / \mathrm{ESH}$ guidelines have been updated, with greater emphasis on the use of SPC in hypertensive patients. In order to provide the RWE on the significance of the triple SPC strategy in Korean patients in accordance with the current hypertension guidelines, the analysis of the results of the present study were based on the $2018 \mathrm{KSH}$ and $2018 \mathrm{ESC} / \mathrm{ESH}$ guidelines. This involved adjustment of age and target BP of the elderly group.

In this study, the most common AEs and ADRs included dizziness, headache, hypotension and arthralgia. A similar AE profile was observed in TRINITY study where dizziness (9.9\%), peripheral edema (7.7\%), and headache $(6.4 \%)$ occurred most frequently [10]. No additional safety issue was identified that requires reassessment of the safety of OM/AML/HCTZ.

This study has the inherent limitations associated with multicenter, non-comparative, observational study design. The presence of missing data was unavoidable due to large datasets. However, the current study has strengths in its prospective study design and in that a large number of more than 3000 hypertensive patients in Korea were evaluated in a real clinical setting over a longer period than previous studies [11]. Therefore, this study contributed to establishing a rationale for use of the triple SPC in Korean patients and has proven that the standard dose of OM/AML/HCTZ is a safe and effective option for the majority of Korean patients with essential hypertension.

Considering previous studies of OM/AML/HCTZ that showed reduced cardiovascular events and mortality by early and long-term BP control, future research may investigate the long-term effectiveness of OM/AML/ HCTZ on cardiovascular outcomes in Korean patients [16-18]. 


\section{Conclusions}

The triple SPC of OM/AML/HCTZ significantly reduced the mean SBP/DBP relative to the baseline in Korean patients with essential hypertension. The BP lowering effects were maintained throughout the 12-month observation period along with high patient adherence and BP control rates. OM/AML/HCTZ was safe and well-tolerated in Korean patients.

\begin{abstract}
Abbreviations
ADRs: Adverse reactions; AEs: Adverse events; AML: Amlodipine; BP: Blood pressure; CVD: Cardiovascular disease; DBP: Diastolic blood pressure; DM: Diabetes mellitus; ESC: European Society of Cardiology; ESH: European Society of Hypertension; HCTZ: Hydrochlorothiazide; KSH: Korean Society of Hypertension; MPR: Medication possession ratio; NRS: Numeric rating scale; OM: Olmesartan; RCTs: Randomized-controlled trials; RD: Renal disease; RWE: Real-world evidence; SBP: Systolic blood pressure; SADRs: Serious adverse reactions; SAEs: Serious adverse events; SPC: Single-pill combination
\end{abstract}

\section{Supplementary Information}

The online version contains supplementary material available at https://doi. org/10.1186/s40885-021-00177-z.

Additional file 1: Supplementary Figure 1. Time course change in the mean SPB/DBP at month 3, 6, 9 and 12 in patients treated only with $\mathrm{OM} / \mathrm{AML} / \mathrm{HCTZ}$. Supplementary Table 1. Changes from baseline in the mean SBP/DBP at month 3, 6, 9 and 12 in patients with risk factors.

Supplementary Table 2. Changes from baseline in the mean SBP/DBP at month 3, 6, 9 and 12 in patients with different age groups. Supplementary Table 3. Participating institutions.

\section{Acknowledgements}

The authors greatly acknowledge the contribution of all the investigators and research coordinators at each participating institution. The authors also thank LSK Global Pharma Services Co., Ltd. for statistical analysis assistance. Authors details

Investigators: Yun Sung Kim (Chosun University Hospital), Seong-III Woo (Inha University Hospital), Kyounghoon Lee (Gacheon University Gil Medical Center), Jung Ho Heo (Kosin University Gospel Hospital), Sang-Ho Park (Soonchunhyang University Cheonan Hospital), Euy Jin Choi (Bucheon St. Mary's Hospital), Sun Ho Hwang (Gwangju Veterans Hospital), Yonh-Gu Chung (Korea University Anam Hospital), Young Jin Youn (Wonju Severance Christian Hospital), Eul-Soon Im (Dongsuwon General Hospital), Jong Sam Baik (Inje University Sanggye Paik Hospital), Nack-Cheon Choi (Gyeongsang National University Hospital), Jin Bae Lee (Daegu Catholic University Medical Center), Kyu-Hyung Ryu (Hallym University Dongtan Sacred Heart Hospital), Ji-Yong Jang (Konkuk University Chungju Hospital), Sung-Ji Park (Samsung Medical Center), Dong-Ick Shin (Chungbuk National University Hospital), Jin Oh Na (Korea University Guro Hospital), Yun Jae Chung (Chung-Ang University Hospital), Su Hyun Kim (St. Carollo Hospital), Jong-Young Lee (Kangbuk Samsung Hospital), Yoon-Sang Oh (Yeouido St. Mary's Hospital), Jong Hwan Choi (Daegu Fatima Hospital), Hye Sun Seo (Soonchunhyang University Bucheon Hospital), Su Kyoung Kwon (Kosin University Gospel Hospital), HyungJun Kim (Dongkang Medical Center), Jin-Sun Park (Ajou University Hospital), Kyoung-Ha Park (Hallym University Sacred Heart Hospital), Kyoung-Soo Lee (Samsung Changwon Hospital), Sung Chang Chung (Dongkang Medical Center), Han-Jin Cho (Pusan National University Hospital), Chang-Min Lee (Dankook University Hospital), Sung-Rae Kim (Bucheon St. Mary's Hospital), Jinkwon Kim (CHA Bundang Medical Center), Hyeong-gyun Oh (Gwangju Veterans Hospital), Ik Seong Park (Bucheon St. Mary's Hospital), Pil-Wook Chung (Kangbuk Samsung Hospital), Sung-Rae Cho (Changwon Fatima Hospital), Hyun-Seung Kang (Seoul National University Hospital), Seung Jin Lee (Soonchunhyang University Cheonan Hospital), Kyungil Park (Dong-A University Hospital), Si Won Lee (Busan Veterans Hospital), Jang-Won Son (Yeungnam University Medical Center), Soo Kyoung Kim (Gyeongsang National University Hospital), Joong-Hwa Chung (Chosun University Hospital), Jin-Soo Byon (Soonchunhyang University Gumi Hospital), Hong Euy Lim (Korea
University Guro Hospital), Bum-Tae Kim (Soonchunhyang University Bucheon Hospital), Yu Jeong Choi (Eulji University Hospital), Soe Hee Ann (Ulsan University Hospital), Sang Min Sung (Pusan National University Hospital), JunHee Lee (Kangdong Sacred Heart Hospital).

\section{Authors' contributions}

ISS, SHI, GHK, SMP, DIC, BKH, CHL, SHL, SPJ, SCL, YHL, DWJ, KTJ, and CJK participated in the enrollment and performed clinical follow-up and data acquisition. ISS, SHI, GHK, SMP, and CJK contributed to the analysis and interpretation of the data. SJR and YJC contributed to data gathering and analysis. ISS wrote the first draft and revised the manuscript. CJK contributed to the review and critical revision of the manuscript. All authors reviewed and approved the final manuscript.

\section{Funding}

This study was funded by Daiichi Sankyo Korea Co., Ltd.

Availability of data and materials

The datasets are not publicly available but are available from the corresponding author upon reasonable request.

\section{Declarations}

\section{Ethics approval and consent to participate}

The study was conducted in accordance with the Declaration of Helsinki and was approved by Institutional Review Board (IRB) and ethics committee at each participating institution. Written informed consent was obtained from all enrolled patients prior to study initiation. The information of IRB approval is included as supplementary material (Table S3).

\section{Consent for publication}

Not applicable.

\section{Competing interests}

All authors received research funds from Daiichi Sankyo Korea Co., Ltd. SJR and YJC are full-time employees of Daiichi Sankyo Korea Co., Ltd. There are no further conflicts of interests to declare.

\section{Author details}

${ }^{1}$ Department of Internal Medicine, Division of Cardiology, Kyung Hee University Hospital at Gangdong, Seoul, Republic of Korea. ${ }^{2}$ Department of Internal Medicine, Division of Cardiology, Bucheon St. Mary's Hospital, College of Medicine, The Catholic University of Korea, Bucheon, Republic of Korea. ${ }^{3}$ Department of Internal Medicine, Division of Cardiology, St. Vincent's Hospital, Suwon, Republic of Korea. ${ }^{4}$ Department of Cardiology, Nowon Eulji Medical Center, Eulji University, Seoul, Republic of Korea. ${ }^{5}$ Division of Cardiology, Heart Center, Gangnam Severance Hospital, Yonsei University College of Medicine, Seoul, Republic of Korea. ${ }^{6}$ Department of Internal Medicine, Division of Cardiology, Veterans Health Service Medical Center, Seoul, Republic of Korea. ${ }^{7}$ Department of Internal Medicine, Division of Cardiology, Pusan National University Yangsan Hospital, Yangsan, Republic of Korea. ${ }^{8}$ Department of Neurology, Kyung Hee University Hospital, Seoul, Republic of Korea. ${ }^{9}$ Department of Neurosurgery, Chonnam National University Hospital, Chonnam National University Medical School, Gwangju, Republic of Korea. ${ }^{10}$ Department of Neurology, Dong-Eui Hospital, Busan, Republic of Korea. ${ }^{11}$ Department of Internal Medicine, Yonsei University College of Medicine, Seoul, Republic of Korea. ${ }^{12}$ Department of Internal Medicine, Division of Cardiology, National Health Insurance Service IIsan Hospital, Goyang, Republic of Korea. ${ }^{13}$ Department of Internal Medicine, Division of Cardiology, Eulji University Hospital, Daejeon, Republic of Korea.

${ }^{14}$ Medical Affairs Department, Daiichi Sankyo Korea Co., Ltd., Seoul, Republic of Korea. ${ }^{15}$ Department of Cardiology, CHA Gangnam Medical Center, CHA University School of Medicine, Seoul, South Korea.

Received: 17 February 2021 Accepted: 22 August 2021

Published online: 01 November 2021

\section{References}

1. Mills KT, Bundy JD, Kelly TN, Reed JE, Kearney PM, Reynolds K, et al. Global disparities of hypertension prevalence and control: a systematic analysis of 
population-based studies from 90 countries. Circulation. 2016;134(6):441-50. https://doi.org/10.1161/CIRCULATIONAHA.115.018912.

2. Lee HY, Shin J, Kim GH, Park S, Ihm SH, Kim HC, et al. 2018 Korean Society of Hypertension Guidelines for the management of hypertension: part IIdiagnosis and treatment of hypertension. Clin Hypertens. 2019;25(1):20. https://doi.org/10.1186/s40885-019-0124-x.

3. Wald DS, Law M, Morris JK, Bestwick JP, Wald NJ. Combination therapy versus monotherapy in reducing blood pressure: meta-analysis on 11,000 participants from 42 trials. Am J Med. 2009;122(3):290-300. https://doi.org/1 0.1016/j.amimed.2008.09.038.

4. Neutel JM. The role of combination therapy in the management of hypertension. Nephrol Dial Transplant. 2006;21(6):1469-73. https://doi.org/1 0.1093/ndt/gfk064.

5. Williams N, Mancia G, Spiering W, Rosei EA, Azizi M, Burnier B, et al. 2018 ESC/ESH guidelines for the management of arterial hypertension: the task force for the management of arterial hypertension of the European Society of Cardiology (ESC) and the European Society of Hypertension (ESH). Eur Heart J. 2018;39(33):3021-104. https://doi.org/10.1093/eurheartj/ehy339.

6. Kim HC, Ihm SH, Kim GH, Kim JH, Kim Kl, Lee HY, et al. 2018 Korean Society of Hypertension guidelines for the management of hypertension: part Iepidemiology of hypertension. Clin Hypertens. 2019;25(1):16. https://doi. org/10.1186/s40885-019-0121-0.

7. Levi M, Pasqua A, Cricelli I, Cricelli C, Piccinni C, Parretti D, et al. Patient adherence to olmesartan/amlodipine combinations: fixed versus extemporaneous combinations. J Manag Care Spec Pharm. 2016;22(3):25562. https://doi.org/10.18553/jmcp.2016.22.3.255.

8. Oparil S, Melino M, Lee J, Fernandez V, Heyrman R. Triple therapy with olmesartan medoxomil, amlodipine besylate, and hydrochlorothiazide in adult patients with hypertension: the TRINITY multicenter, randomized, double-blind, 12-week, parallel-group study. Clin Ther. 2010;32(7):1252-69. https://doi.org/10.1016/j.clinthera.2010.07.008.

9. Kereiakes DJ, Chrysant SG, Izzo JL Jr, Littlejohn T 3rd, Oparil S, Melino M, et al. Long-term efficacy and safety of triple-combination therapy with olmesartan medoxomil and amlodipine besylate and hydrochlorothiazide for hypertension. J Clin Hypertens (Greenwich). 2012;14:149-57.

10. Bramlage P, Fronk EM, Wolf WP, Smolnik R, Sutton G, Schmieder RE. Safety and effectiveness of a fixed-dose combination of olmesartan, amlodipine, and hydrochlorothiazide in clinical practice. Vasc Health Risk Manag. 2014; 11:1-8.

11. Mohan JC, Jain R, Chamle V, Bhargava A. Short term safety and tolerability of a fixed dose combination of olmesartan, amlodipine and hydrochlorothiazide. J Clin Diagn Res. 2015;9(8):OC10-3. https://doi.org/10. 7860/JCDR/2015/14054.6366

12. Park SJ, Rhee SJ. Real-world effectiveness and safety of a single-pill combination of olmesartan/amlodipine/hydrochlorothiazide in Korean patients with essential hypertension (RESOLVE): a large, observational, retrospective, cohort study. Adv Ther. 2020;37(8):3500-14. https://doi.org/1 0.1007/s12325-020-01404-z.

13. Volpe M, Gallo G, Tocci G. Is early and fast blood pressure control important in hypertension management? Int J Cardiol. 2018;254:328-32. https://doi. org/10.1016/j.ijcard.2017.12.026

14. Ishisaka DY, Jukes T, Romanelli RJ, Wong KS, Schiro TA. Disparities in adherence to and persistence with antihypertensive regimens: an exploratory analysis from a community-based provider network. J Am Soc Hypertens. 2012;6(3):201-9. https://doi.org/10.1016/j.jash.2012.02.004.

15. Panjabi S, Lacey M, Bancroft T, Cao F. Treatment adherence, clinical outcomes, and economics of triple-drug therapy in hypertensive patients. J Am Soc Hypertens. 2013;7(1):46-60. https://doi.org/10.1016/j.jash.2012.11.001.

16. Julius S, Kjeldsen SE, Weber M, Brunner HR, Ekman S, Hansson L, et al. VALUE trial group Outcomes in hypertensive patients at high cardiovascular risk treated with regimens based on valsartan or amlodipine: the VALUE randomised trial. Lancet. 2004;363(9426):2022-31. https://doi.org/10.1016/ S0140-6736(04)16451-9

17. ALLHAT Officers and Coordinators for the ALLHAT Collaborative Research Group. Major outcomes in high-risk hypertensive patients randomized to angiotensin-converting enzyme inhibitor or calcium channel blocker vs diuretic: the Antihypertensive and Lipid-Lowering Treatment to Prevent Heart Attack Trial (ALLHAT). JAMA. 2002;288(23):2981-97. https://doi.org/10.1 001/jama.288.23.2981.

18. Dahlof B, Sever PS, Poulter NR, Wedel H, Beevers DG, Caulfield M, et al. Prevention of cardiovascular events with an antihypertensive regimen of amlodipine adding perindopril as required versus atenolol adding bendroflumethiazide as required, in the Anglo-Scandinavian Cardiac Outcomes Trial-Blood Pressure Lowering Arm (ASCOT-BPLA): a multicentre randomised controlled trial. Lancet. 2005;366(9489):895-906. https://doi. org/10.1016/50140-6736(05)67185-1.

\section{Publisher's Note}

Springer Nature remains neutral with regard to jurisdictional claims in published maps and institutional affiliations.
Ready to submit your research? Choose BMC and benefit from:

- fast, convenient online submission

- thorough peer review by experienced researchers in your field

- rapid publication on acceptance

- support for research data, including large and complex data types

- gold Open Access which fosters wider collaboration and increased citations

- maximum visibility for your research: over $100 \mathrm{M}$ website views per year

At BMC, research is always in progress.

Learn more biomedcentral.com/submissions 